\title{
Desdobramento da Qualidade no Desenvolvimento de Filmes Flexíveis para Embalagens
}

\author{
Paulo A. Cauchick Miguel \\ Faculdade de Engenharia Mecânica e de Produção, UNIMEP \\ Mônica Telfser, Alexandre Maruca, Antônio Gallonetti, Aquiles Saracura, Luis Martins, \\ Mário Hori, Paulo Ribeiro, Roni de Campos, Tanyze Marconato, Valmir Mora \\ Votocel Filmes Flexíveis Ltda.
}

\begin{abstract}
Resumo: Um dos métodos atuais importantes para o desenvolvimento de produtos é o QFD (Quality Function Deployment ou Desdobramento da Função Qualidade). O QFD consiste em um processo sistemático para traduzir a opinião do cliente em requisitos técnicos e operacionais, mostrando e documentando as informações na forma de matrizes. O método objetiva enfocar os itens mais importantes, proporcionando um mecanismo para alcançar vantagens competitivas no processo de desenvolvimento. Este trabalho destaca o conceito do QFD, citando uma aplicação em uma empresa que produz filmes flexíveis de polipropileno (BOPP). O trabalho apresenta alguns aspectos da introdução do método na empresa, apontando as dificuldades encontradas, resultados e benefícios decorrentes da implantação. Dentre os benefícios gerados, destacamse a importância do papel do QFD como um método para sistematizar o processo de desenvolvimento, bem como outros benefícios para a equipe, tais como melhoria nas habilidades de planejamento, disseminação do conhecimento e melhoria de comunicação entre as áreas funcionais.
\end{abstract}

Palavras chave: Desdobramento da qualidade, QFD, filmes de BOPP, desenvolvimento de produto.

\section{Quality Deployment in the Development of Flexible Films for Packaging}

Abstract: One of the important methods for product development is QFD (Quality Function Deployment). QFD consists of a systematic process to translate customer's opinion into technical and operational requirements, pointing out and recording information by using matrices. The method aims at focusing in the most important items, resulting in a way to achieve competitive advantages in the development process. This paper highlights the concept of QFD and reports the introduction of QFD in a company which produces polypropylene flexible (BOPP) films. The work discusses the way QFD was introduced in the company and points out the experienced difficulties, achieved results as well as the benefits from the implementation. Among those benefits, it can be highlighted the QFD role as a method to systematise the development process, besides other teamwork benefits, such as improvement of planning abilities, knowledge dissemination, and better communication between functional areas.

Keywords: QFD, quality deployment, BOPP, flexible films, product development.

\section{Introdução}

O QFD (Quality Function Deployment ou Desdobramento da Função Qualidade) consiste em um processo sistemático para traduzir os requisitos dos clientes em características da qualidade dos produtos, possibilitando documentar as informações necessárias para o processo de desenvolvimento. O método objetiva enfocar os itens mais importantes, tanto do ponto de vista dos clientes quanto da empresa, proporcionando um mecanismo para alcançar vantagens competitivas no desenvolvimento de produto. O método foi desenvolvido no Japão a partir do final dos anos 60 , tendo sido conhecido no ocidente, na forma que é hoje utilizado, no ano de 1972. O QFD foi introduzido nos EUA e Europa (mais especificamente na Itália) no início da década de 80. A história do QFD no Brasil é um pouco mais recente, com cerca de dez anos. Foi citado pela primeira vez no país, por um dos seus criadores, Yoji Akao, em uma conferência sobre Controle da Qualidade realizada no Rio de Janeiro ${ }^{[1]}$. A partir de 1990 começou a ser utilizado por muitas empresas. Dentre as aplicações relatadas no país, podem ser citados casos de implantação na indústria automotiva ${ }^{[2-5]}$, empresas siderúrgicas ${ }^{[6]}$ e setores de serviços, tais como em empresas do setor hoteleiro ${ }^{[7]}$, academia de ginástica ${ }^{[8]}$ dentre outros ${ }^{[9,10]}$. Desde as primeiras aplicações no Brasil, têm sido relatados casos de implantação bem sucedida em empresa do setor de alimentos ${ }^{[11-13]}$, destacando-se também aplicação no desenvolvimento de embalagens ${ }^{[14]}$.

Como exemplo de aplicação do QFD na área de polímeros em outras partes do mundo, pode ser citado tra-

Autor para Correspondência: Paulo A. C. Miguel, NGQM-FEMP-UNIMEP, Rodovia SP 306, km 1, CEP: 13450-000, Santa Bárbara d'Oeste, SP. E-mail: pamiguel@unimep.br 
balho de Yeung e Lau ${ }^{[15]}$. O artigo apresenta uma aplicação do QFD em conjunto com outras técnicas (tais como o método de Tagucchi para planejamento de experimentos e ANOVA - Análise de Variância), no projeto de moldes de injeção para termoplásticos, utilizando um software de simulação CAE (Computer Aided Engineering). Nesse estudo de caso, um arranjo ortogonal foi utilizado no experimento e sete parâmetros do processo de injeção foram testados, utilizando a ANOVA para otimização do processo de injeção de uma peça plástica circular. Neste caso, o QFD foi aplicado para priorizar algumas etapas do trabalho, buscando alcançar a satisfação dos clientes.

Nesse contexto, o propósito deste trabalho é apresentar o conceito do QFD e ilustrar sua implantação em uma empresa da área de polímeros no Brasil, que produz filmes flexíveis de polipropileno, utilizados em embalagens de alimentos, rótulos, etiquetas, dentre outras aplicações. O trabalho destaca a seqüência para implantação, descrevendo sobre suas etapas e cronograma. Também são apontadas as dificuldades encontradas durante a implantação, os resultados alcançados até o momento e os benefícios decorrentes do uso do método.

\section{Conceito do QFD}

O QFD surgiu a partir das necessidades de garantir a qualidade no desenvolvimento do produto, ou seja, na fase de projeto, de forma a garantir esse projeto na pré-produção, isto é, antes do produto entrar em fabricação.

A fim de que de que se possa ter garantia da qualidade no projeto, o QFD possibilita traduzir os requisitos e necessidades dos clientes (qualidades exigidas) em características da qualidade, ou seja, em atributos e especificações do produto, buscando o desdobramento da qualidade do projeto para o produto acabado através do uso de matrizes. Essas matrizes contém as informações e dados necessários para que a qualidade seja obtida. Entretanto, as informações das matrizes devem ser colocadas em prática. Nesse sentido, o QFD também atua como uma forma de comunicar sistematicamente a informação relacionada com a qualidade e de explicitar ordenadamente o respectivo trabalho (função) para colocar em prática essas informações.

Dessa forma, observa-se que o QFD contempla esses dois aspectos fundamentais: o desdobramento da qualidade e o desdobramento da função. O desdobramento da qualidade é tradução das qualidades exigidas pelos clientes em características da qualidade do produto, processo, e produção, representado por um conjunto de matrizes. O desdobramento da função, por sua vez, significa o desdobramento do trabalho, ou seja, a seqüência, o registro e documentação de todas as funções operacionais e trabalho necessários para obtenção da qualidade desdobrada nas matrizes elaboradas. Na realidade, o que é denominado comumente de QFD é somente o Desdobramento da Qualidade, enquanto que o desdobramento da função é denominado de "QFD no sentido restrito" (tradução feita de narrowly defined $Q F D)^{[16,17]}$. A combinação dessas duas partes forma então o QFD Amplo, ilustrado na Figura 1.

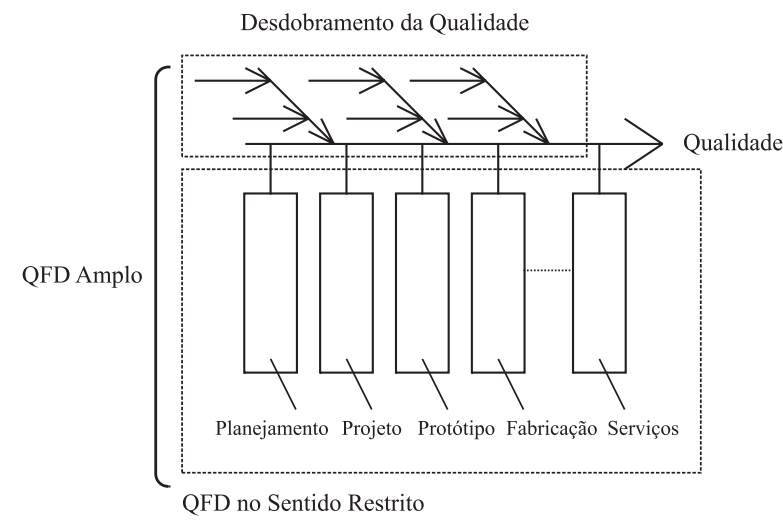

Figura 1. Estrutura do QFD Desenvolvida por $\mathrm{Akao}^{[17]}$.

Como citado anteriormente, o desdobramento da qualidade compreende a conversão dos requisitos dos usuários nas características substitutivas (características da qualidade), ou seja, aquelas características que substituirão as demandas dos clientes, determinando então a qualidade no projeto de um produto completo ${ }^{[16,17]}$. Se esse produto for específico da indústria de montagem, por exemplo, será usualmente formado por um conjunto e sub-conjuntos. Nesse caso, a qualidade deve ser sistematicamente desdobrada para cada componente do produto do conjunto e subconjuntos e, sucessivamente, para os processos e seus elementos que os compõem, considerando também as relações entre eles. De modo análogo, se o produto é pertencente a indústria de processos, a qualidade deverá ser desdobrada para as matérias-prima, aditivos e processos de obtenção do produto. O QFD no sentido restrito por sua vez pode ser obtido pelo desdobramento sistemático das funções de trabalho (planejamento, projeto, produção, serviços, etc.) e operações que contribuem para a qualidade nas etapas sucessivas para obtenção do produto, traduzindo-se no trabalho necessário para obter a qualidade. A matriz mais comum na utilização do QFD, e de onde se inicia todo o processo de desdobramento, é aquela que relaciona as qualidades exigidas (requisitos dos clientes) com as características da qualidade (especificações) do produto. Essa matriz é ilustrada na Figura 2.

As qualidades exigidas expressam os requisitos, necessidades e expectativas dos clientes, traduzidos pelo que é denominado de $\mathrm{Voz}$ do Cliente (VoC - Voice of Customer). A essas qualidades exigidas são atribuídos grau de importância, geralmente em uma escala Lickert de 5 pontos (variando de 1 a 5 , sendo 1 sem importância e 5 muito importante). Em

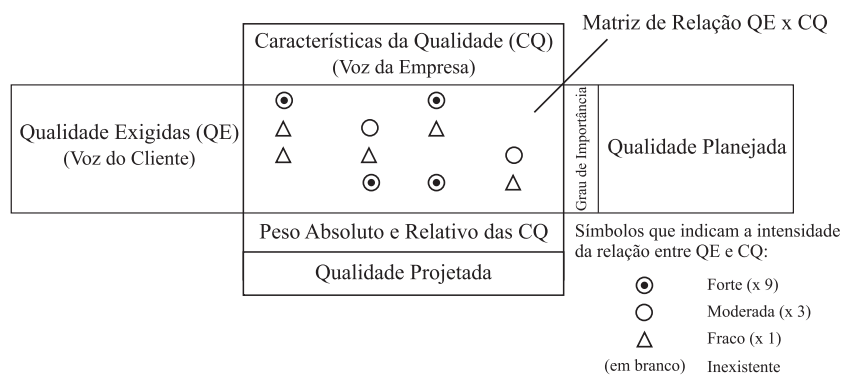

Figura 2. Qualidades Exigidas versus Características da Qualidade (adaptado de Miguel ${ }^{[18]}$ ). 
seguida, é feita a qualidade planejada que compreende uma avaliação (feita pelo cliente) sobre o desempenho do produto da empresa e dos produtos concorrentes (escala de 1 a 5). A partir dessa comparação, determina-se a qualidade planejada (também em uma escala de 1 a 5) e a taxa de melhoria (qualidade planejada dividida pelo desempenho atual do produto) $\mathrm{e} o$ argumento de vendas. $\mathrm{O}$ valor do argumento de vendas é uma ponderação, que pode ser de 1, 1,2 ou 1,5, sobre quão estratégica é cada qualidade exigida. Finalmente, calcula-se o peso absoluto e relativo (peso absoluto normalizado) de cada qualidade exigida pela multiplicação desses fatores (grau de importância $\mathrm{x}$ taxa de melhoria $\mathrm{x}$ argumento de vendas).

As características da qualidade correspondem aos atributos do produto que tem como objetivo atender as qualidades exigidas, ou seja, correspondem às especificações. $\mathrm{Na}$ parte central da matriz tem-se uma análise do impacto que cada característica da qualidade tem nas qualidades exigidas, expressos pelo grau de relação de intensidade (forte, moderado, fraco ou inexistente, geralmente numa escala, respectivamente, de 9, 3,1 e 0). Através da multiplicação do peso relativo de cada qualidade exigida por essa relação de intensidade e somando-se na coluna de cada característica da qualidade tem-se como resultado o peso absoluto de cada uma. Essa importância (peso absoluto) pode ser expressa por:

$$
\mathrm{PCQ}_{\mathrm{j}}=\sum_{\mathrm{i}=1}^{\mathrm{n}} \mathrm{PQE}_{\mathrm{i}} \mathrm{R}_{\mathrm{ij}} \quad \forall_{j}, j=1, \ldots, m
$$

Onde:

$P C Q_{j}=$ peso absoluto da j-ésima característica da qualidade; $P Q E_{i}=$ peso relativo (ou absoluto) da i-ésima qualidade exigida;
$\mathrm{R}_{\mathrm{ij}}=$ relação de intensidade entre a j-ésima característica da qualidade e a i-ésima qualidade exigida;

$n=$ número de qualidades exigidas;

$m=$ número de características da qualidade.

Ter-se-á como resultado quais serão as características da qualidade que devem ser priorizadas no desenvolvimento, a partir do maior peso absoluto (ou peso relativo, que corresponde ao absoluto normalizado). É também importante destacar que a decisão sobre quais características da qualidade devem ser priorizadas não é feita somente baseada nesse resultado. Além disso, também é realizada uma comparação entre o produto da empresa com o(s) produto(s) concorrente(s), neste caso realizado pela própria engenharia utilizando as grandezas correspondentes às especificações. O objetivo é verificar se o produto da empresa tem um desempenho superior naquela dada característica da qualidade. A partir dessa análise, tem-se como resultado a qualidade projetada, isto é, o valor de especificação que deverá ser adotado e perseguido no projeto.

A Figura 3 apresenta um exemplo de uma matriz da qualidade para o desenvolvimento de um frasco para embalagem. Nota-se pelo resultado da qualidade projetada, que as características da qualidade "encaixe da tampa com flip-top" e "escoamento" são aquelas que devem ser priorizadas, pois, além resultarem no maior peso relativo (respectivamente 36 e 34,6\%), também têm desempenho inferior ao concorrente, verificado pela mensuração de cada característica da qualidade da empresa ("produto atual/protótipo") com o concorrente ("G\&G").

Posteriormente, outras matrizes também podem ser desenvolvidas, a partir dessa mesma lógica. Como exemplos dessa matrizes podem ser citadas: características da qualidade $\mathrm{x}$ componentes (tampa, frasco e rótulo), componentes $\mathrm{x}$

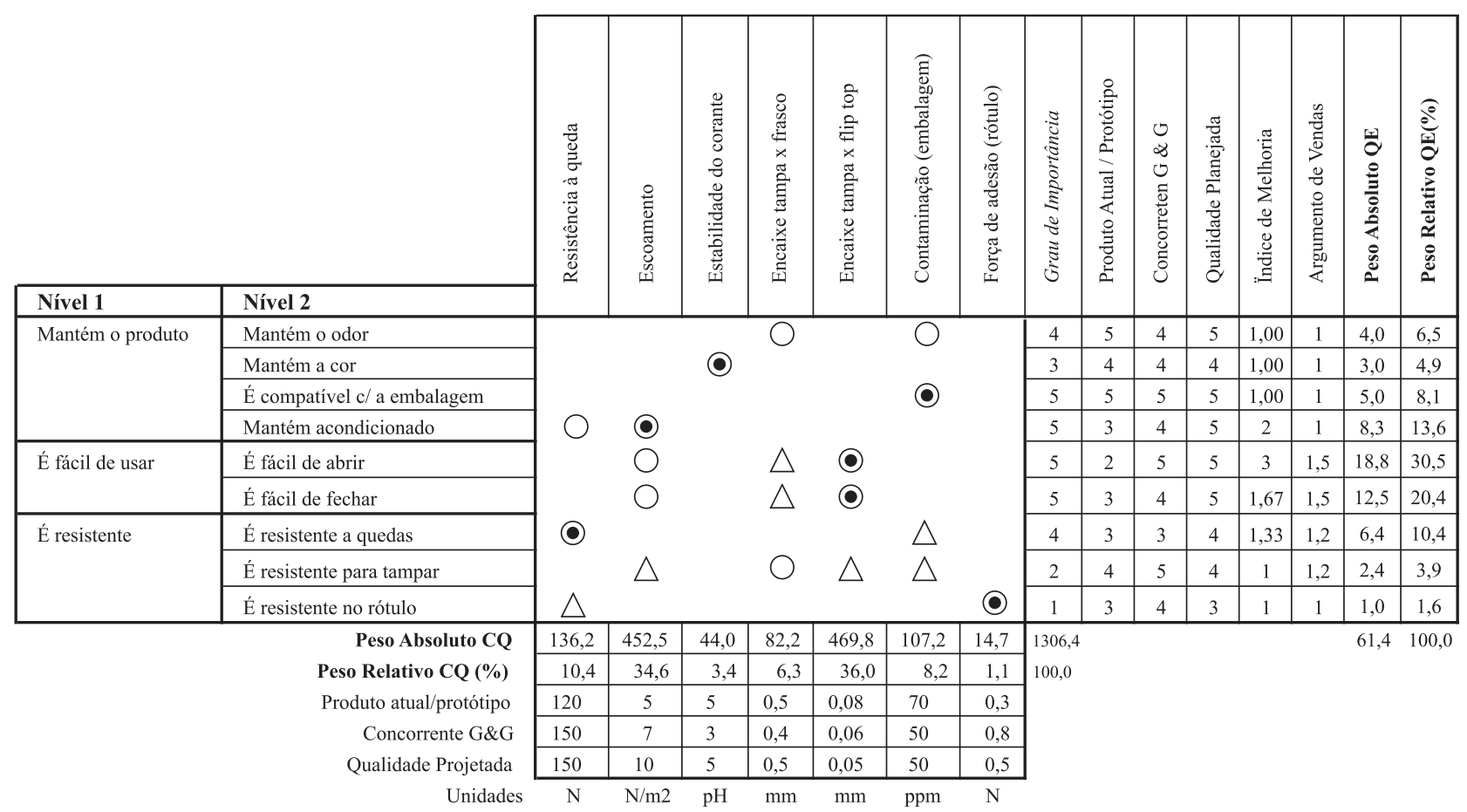

Figura 3. Exemplo de Matriz para Desenvolvimento de um Frasco (Desenvolvido pelo Autor). 
parâmetros de controle dos processos, componentes x matéria-prima, dentre outras.

A seguir, é descrito uma implantação do método no desenvolvimento de filmes flexíveis para embalagens, ilustrando o uso do QFD nesse tipo de aplicação.

\section{Aplicação do QFD no Desenvolvimento de Filmes de BOPP para Embalagens}

Filmes de BOPP são filmes de polipropileno orientado biaxialmente (biaxially oriented polypropylene), obtidos através de um processo de transformação da resina granulada de polipropileno. O polipropileno provêm do propeno, que por sua vez é subproduto da nafta, esta obtida após destilação fracionada do petróleo. A Figura 4 ilustra o processo de fabricação para obtenção do filme de BOPP, que compreende basicamente as seguintes etapas ${ }^{[19]}$ : extrusão, para a fusão do polipropileno; formação, onde ocorre a formação e resfriamento do filme de polipropileno; estiramento longitudinal que promove, através de um estiramento mecânico, a orientação das cadeias de polipropileno na direção longitudinal do filme; estiramento transversal que, a exemplo da etapa anterior, promove a orientação por meio de estiramento mecânico, porém na direção transversal, resultando assim na película de polipropileno orientada biaxialmente; tração e tratamento da película orientada e embobinamento.

Em função da orientação das cadeias de polipropileno, a película resultante deste processo possui propriedades de barreira, rigidez e resistência mecânica muito superiores as de um filme de polipropileno não orientado com espessura equivalente. Essas propriedades permitem sua utilização em espessuras muito inferiores àquelas de um filme de polipropileno não orientado, possibilitando o uso desse filme em várias aplicações em embalagens de produtos em geral (alimentos e não alimentos), bem como aplicações industriais (fitas adesivas, etiquetas, plastificação, etc.). Nesse sentido, essas propriedades e conseqüentes possibilidades de aplicação tornam esse tipo de filme muito atrativo, tanto do ponto de vista financeiro, como de adequação técnica e mercadológica $^{[19]}$.

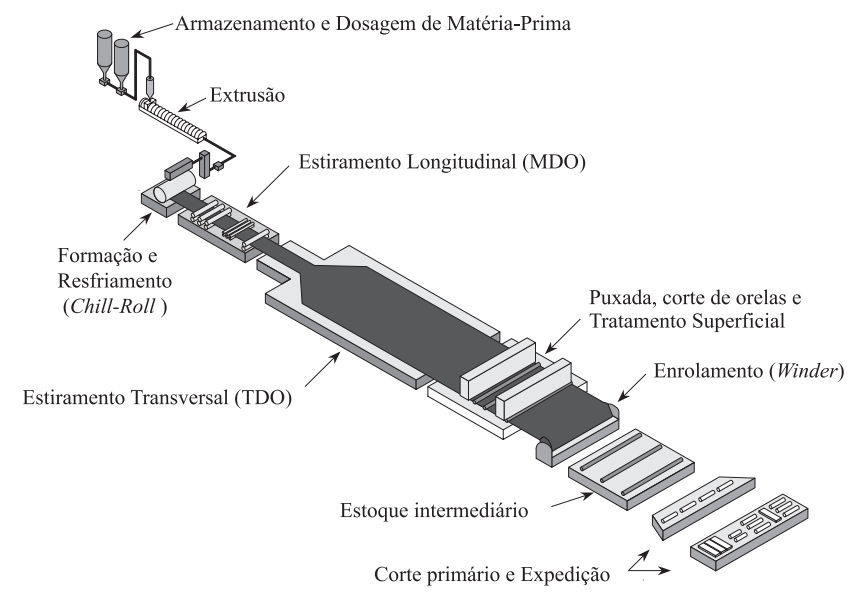

Figura 4. Processo de Fabricação de Filmes de BOPP.

\section{Implantação do Método}

A definição da seqüência de implantação foi baseada no trabalho de Cheng et al. ${ }^{[16]}$, sendo dividida em cinco etapas:

1) definição das metas para desenvolvimento do projeto;

2) desenvolvimento do modelo conceitual;

3) elaboração da matriz da qualidade;

4) elaboração das outras matrizes do modelo conceitual;

5) definição do plano de ação.

Essas etapas são descritas a seguir.

\section{Definição das Metas de Desenvolvimento do Projeto}

As metas do projeto do produto englobam um conjunto de objetivos que se espera alcançar com o desenvolvimento do projeto. Basicamente, servem para definir o que se espera alcançar com o projeto. As metas devem considerar aspectos comerciais (de mercado), além das dimensões de qualidade, tecnologia, confiabilidade e custo, conforme sugerido por Akao ${ }^{[17]}$. Exemplos de metas do projeto piloto são apresentadas a seguir (entre parênteses encontra-se a classificação de cada uma das metas apresentadas, conforme recomendado na literatura): ${ }^{[16-18]}$

- Redução no número de não conformidades e reclamações de mercado (qualidade).

- Otimizar os atributos básicos do produto (tecnologia).

- Identificar aspectos que agregam custo, mas não agregam valor (custo).

- Aumentar base de clientes (mercado).

- Padronização da fabricação do produto em todas as linhas (confiabilidade).

\section{Desenvolvimento do Modelo Conceitual}

O modelo conceitual representa o conjunto de matrizes de um dado desenvolvimento, isto é, o modelo estabelece as relações de causa-efeito entre os elementos integrantes do desenvolvimento tais como especificações, parâmetros de controle do processo, matéria-prima, e outros. Segundo Cheng et al. ${ }^{[16]}$, esse sequenciamento representa o "caminho" pelo qual o desenvolvimento deve percorrer para que se alcance as metas do projeto do produto. Também significa o entendimento sobre a lógica que norteia a fabricação. Sua utilidade está no planejamento do conjunto de matrizes que deverá ser desenvolvido e utilizado.

\section{Elaboração da Matriz da Qualidade}

A matriz da qualidade envolve a relação entre as qualidades exigidas com as características da qualidade. Também inclui a atribuição do grau de importância das qualidades exigidas e análise do produto da empresa e dos produtos dos concorrentes (ambas feitas pelo cliente), definição da qualidade planejada, cálculo do índice de melhoria, definição do argumento de vendas e cálculo do peso absoluto e peso relativo das qualidades exigidas. A partir da relação entre as qualidades exigidas e características da qualidade e do resultado do peso relativo das qualidades exigidas, é calculado o peso 


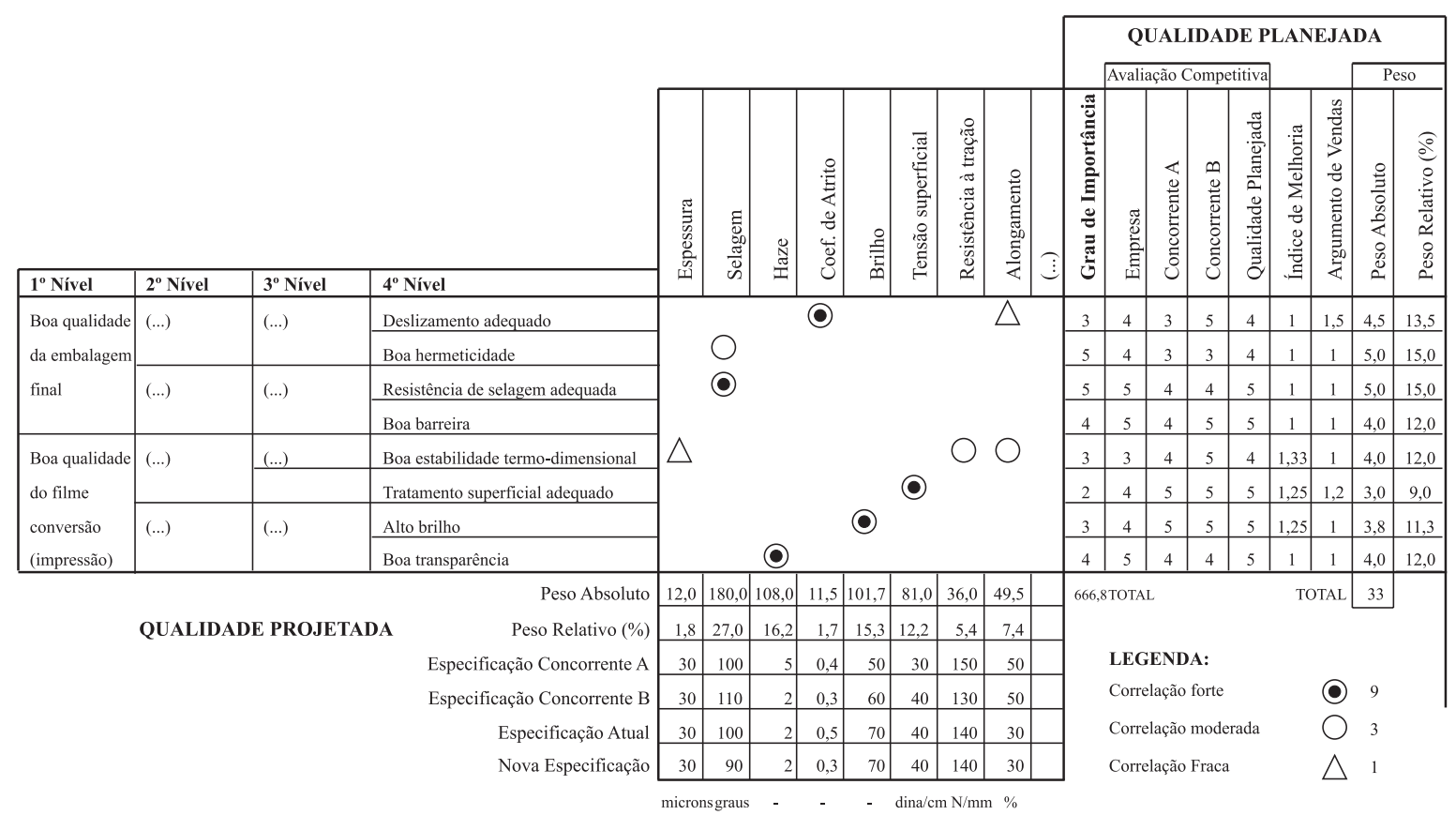

Figura 5. Qualidade Exigida x Características da Qualidade (parcial com valores simulados).

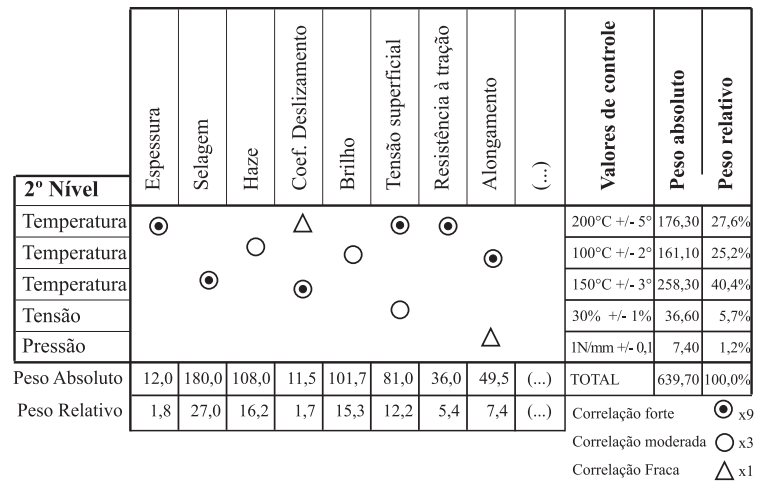

Figura 6. Características da Qualidade x Parâmetros de Processo (parcial com valores simulados).

absoluto e peso relativo das características da qualidade, conforme explanado anteriormente. Além disso, é feita uma análise comparativa entre as características da qualidade do produto da empresa e dos produtos concorrentes (benchmarking de produto). Finalmente, faz-se o estabelecimento da qualidade projetada, ou seja, a definição dos valores alvo ou especificação de cada uma das características, baseado na priorização fornecida pelo peso relativo de cada uma delas e análise das especificações (características da qualidade) do produto da empresa em comparação aos concorrentes. Um exemplo de parte da matriz da qualidade desenvolvida é mostrado na Figura 5.

\section{Elaboração de Outras Matrizes}

Outras matrizes do modelo conceitual também foram desenvolvidas. Essas matrizes definem os parâmetros de controle do processo, com seus respectivos valores e faixas de variação, bem como parâmetros relativos à matéria prima utilizada. A matéria-prima engloba o polipropileno e aditivos que possibilitam obter as propriedades funcionais desejáveis. A Figura 6 mostra um exemplo de uma das matrizes.

\section{Definição do Plano de Ação}

A Figura 7 apresenta o Plano de Ação decorrente do desenvolvimento das matrizes. Para efeito do uso do QFD, as ações que mais interessam são aquelas decorrentes da melhoria de projeto do produto. No entanto, boa parte das ações mostradas na figura são de melhoria de processo ou de produção. É importante destacar que, apesar da identificação desses tipos de ação não ser objetivo principal do QFD, estas foram decorrentes da implantação, podendo ser consideradas como benefícios da sua introdução.

\section{Resultados da Implantação}

Um acompanhamento das reuniões foi realizado pelos pesquisadores no sentido de fornecer apoio técnico, estabelecendo a seqüência do trabalho e, quando necessário, prestando esclarecimentos sobre a metodologia do QFD. Sua atuação também foi importante no sentido de centrar o desenvolvimento do trabalho e a troca de idéias, visando eliminar possíveis divergências.

Ohfuji, Ono e Akao ${ }^{[20]}$ recomendam uma equipe de 5 a 6 membros, pois um número muito superior pode gerar muitas discussões e uma quantidade inferior pode sobrecarregar a equipe. A quantidade de membros da equipe ficou, em média, com 8 participantes nas reuniões realizadas, portanto, não muito acima do número recomendado na literatura.

Com relação a quantidade de horas de trabalho nas reuniões, foi previsto inicialmente que os membros da equipe dedicariam $20 \%$ de seu tempo nesse projeto e o coordenador da equipe $50 \%$. Na realidade, embora tenha sido previsto 5,5 horas por dia de reunião foram despendidas, em média, aproximadamente 4 horas por reunião, totalizando uma carga de trabalho no projeto de pouco mais de 180 horas, eqüivalendo a quase uma jornada mensal de trabalho. 


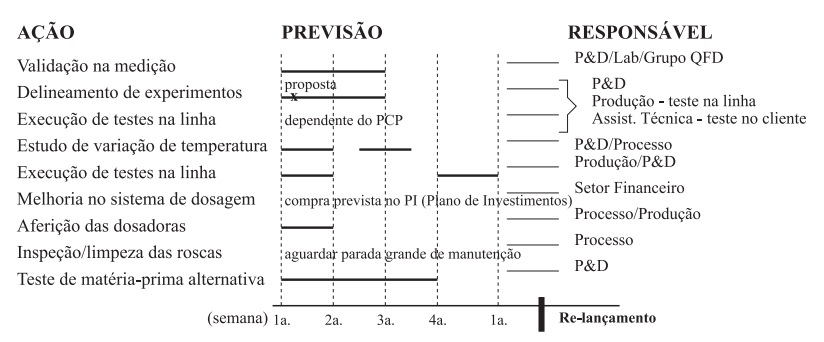

Figura 7. Plano de Ação Decorrente do Desenvolvimento.

O entendimento, a partir da experiência com esse projeto, é que ele efetivamente termina quando todas as especificações (projeto, processo e matéria-prima) estão definidas e o projeto é registrado, ou seja, é escrito o histórico para gerar o aprendizado organizacional e apresentado para outras áreas da empresa. $\mathrm{Na}$ verdade, o projeto termina na especificação, mas um representante da área de processo ou da produção deve monitorar/ acompanhar o produto na produção e no mercado.

\section{Dificuldade de conciliar agenda de reuniões com clientes}

Essa dificuldade pode ser considerada como comum, uma vez que, além de ser necessário consultar vários clientes, é preciso adequar os compromissos de ambas organizações, cliente e fornecedor. Essas visitas foram necessárias para levantar a Voz do Cliente e também para conduzir pesquisas quantitativas (atribuição do grau de importância e avaliação do produto da empresa e do produto dos concorrentes). Foram visitados, no total, 5 clientes, destacando que esse tempo não está sendo considerado no total de horas citado no tópico anterior. Os critérios de seleção desses clientes foram: volume de produção e importância estratégica desses clientes no mercado. Com relação à avaliação de produtos dos concorrentes, foram avaliados 4 empresas concorrentes.

\section{Desconhecimento sobre a metodologia}

Esta também foi uma dificuldade que foi contornada através de alguns "mini-treinamentos" ministrados durante as reuniões para esclarecimento de dúvidas e revisão dos passos para implementação que foram delineados (sem muitos detalhes) em um treinamento de um dia, realizado no início.

\section{Escolha do produto}

A escolha de um produto existente sem dúvida trouxe diversos benefícios, por se tratar de um assunto que a grande maioria do grupo tinha domínio. Entretanto, por ser um projeto de melhoria (produto existente), o grupo fez alguns préconceitos sobre parte das discussões, como por exemplo nas correlações nas matrizes, desfavorecendo a criatividade neces- sária para o desenvolvimento de um projeto. Dessa forma, ocorreu a possibilidade de restringir o potencial de desenvolvimento, uma vez que, em alguns casos, houve pré-julgamento sobre os assuntos tratados. Outro dado importante é que, aparentemente, quando se trata de um produto novo, lança-se um desafio para a equipe. Isso pode ser demonstrado, pois vem ocorrendo na condução de projetos novos utilizando o QFD (ver tópico "Multiplicação do QFD”, adiante nesse trabalho).

\section{Benefícios Alcançados até o Momento}

Apesar das dificuldades relatadas acima, a introdução do QFD como meio de sistematizar o processo de desenvolvimento trouxe muitos benefícios para os participantes. Dentre esses benefícios, destacam-se:

- Desenvolvimento das habilidades de planejamento;

- Disseminação e nivelamento do conhecimento;

- Melhoria de comunicação entre as pessoas e áreas funcionais;

- Visibilidade das informações e registro do know how da empresa.

\section{Desenvolvimento de habilidades de planejamento}

Esse benefício foi alcançado devido ao exercício prático de planejamento que o QFD proporciona. Notadamente, grande parte das empresas não consegue planejar adequadamente, devido a carga de trabalho do dia a dia que, não necessariamente, proporciona a chance de realizar um planejamento preciso. Adicionalmente, as habilidades de planejamento necessitam ser constantemente desenvolvidas. Nesse sentido, a implantação da metodologia por meio do projeto piloto contribuiu significativamente para que essas habilidades fossem desenvolvidas e incorporadas, mesmo ainda que não totalmente, ao processo de desenvolvimento de produto, uma vez que essas habilidades de planejamento estão, de certa forma, ainda restritas na empresa.

\section{Disseminação e nivelamento do conhecimento}

Esse é um dos benefícios reais que o QFD proporciona $^{[17]}$. Nessa primeira fase, ou seja, no desenvolvimento do projeto piloto, já foi possível notar que o conhecimento foi disseminado, apesar de ser difícil quantificar com precisão. Algumas áreas funcionais, que não têm no seu cotidiano oportunidade de interação mais profunda com o desenvolvimento de produto (por exemplo a área de produção), tiveram essa experiência com maior intensidade. Na implantação

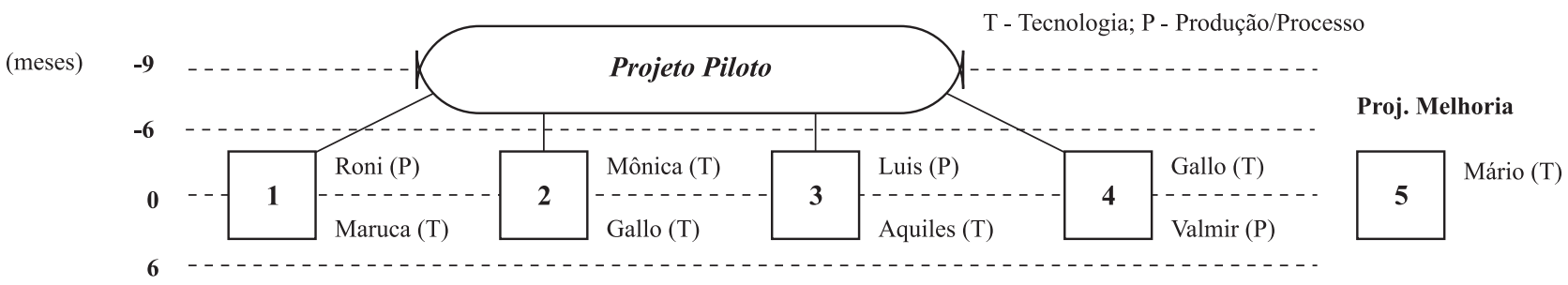

Figura 8. Processo de Difusão para Outros Projetos. 


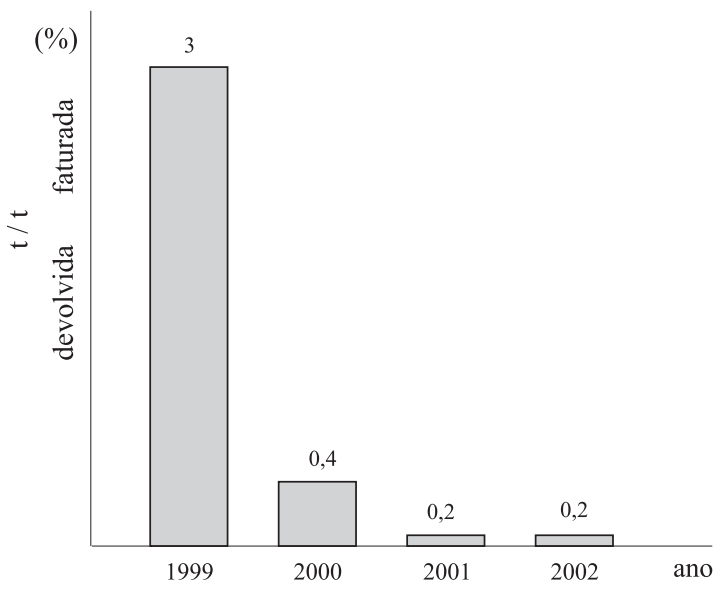

Figura 9. Devoluções - Projeto Piloto.

realizada, em função da equipe ser composta por membros com diferentes tempos de experiência, o nivelamento do conhecimento também foi favorecido.

\section{Melhoria de comunicação entre áreas funcionais}

Obviamente, esse benefício é favorecido em função do trabalho em equipe. Entretanto, como o QFD, pela sua natureza, deve ser conduzido por uma equipe e, geralmente, envolvendo grande diversificação de áreas funcionais, esse ponto positivo ocorre quase que naturalmente.

\section{Visibilidade das informaçōes e registro do know how da empresa}

Esse benefício pode ser considerado como inerente ao QFD, uma vez que é um dos seus princípios básicos, conforme citado por Cheng et a ${ }^{[16]}$. A visibilidade é o segundo princípio, que inclui a pluralização. É um princípio que permeia todas as fases do QFD: na confecção das tabelas, das matrizes, do modelo conceitual e dos padrões de trabalho ${ }^{[16]}$. Isso pode ser realmente notado tanto pela equipe quanto por outras áreas funcionais da empresa que estão familiarizando-se com o método.

\section{Multiplicação do QFD}

Após a conclusão do projeto piloto, a fase atual é a multiplicação do uso do QFD para outros projetos. A Figura 8 ilustra o processo difusão para 4 novos projetos e um de melhoria com o uso do QFD, incluindo a determinação das pessoas que serão os multiplicadores em cada projeto.

\section{Melhorias nos Filmes de BOPP Desenvolvidos com 0 Auxílio do QFD}

Um ponto importante a ser considerado, é a mudança que o uso do QFD como ferramenta de planejamento traz para o processo de desenvolvimento. Além dos fatores organizacionais que a aplicação do método proporciona, como no caso em que o conhecimento de engenharia é retido de uma forma sistemática, para que possa ser resgatado em desenvolvimentos futuros, o método proporciona o desenvolvimento de produtos mais robustos e que estão em maior consonância

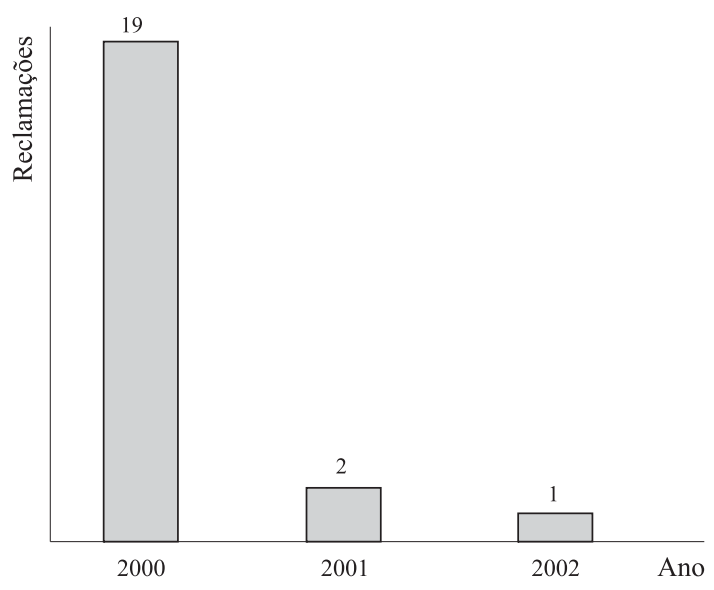

Figura 10. Reclamações - Projeto 4 .

com os clientes. Essa afirmação pode ser ilustrada através dos resultados apresentados nas Figuras 9 e 10. A Figura 9 mostra a redução no índice de devolução do produto do projeto piloto. No caso da Figura 10, esta mostra a redução no número de reclamações para o Projeto 4, citado na Figura 8.

\section{Conclusões}

Os benefícios alcançados até o momento podem ser considerados como satisfatórios. Algumas oportunidades de melhoria puderam ser identificadas, como o registro da experiência, que foi feito pela confecção de um Manual de QFD para a empresa na forma impressa e eletrônica (Intranet).

As avaliações realizadas pela alta direção da empresa (duas) e pelos pesquisadores (cinco) ao longo da implantação auxiliaram a realizar correções de percurso o que também deve contribuir para que os próximos projetos, que envolvem, efetivamente, o desenvolvimento de novos produtos sejam mais eficazes e eficientes. A próxima fase do trabalho é direcionada à conclusão dos 4 novos projetos em andamento com aplicação do QFD.

\section{Agradecimentos}

Os autores agradecem algumas pessoas, sem as quais o desenvolvimento desse trabalho não seria possível. Primeiramente, ao Sr. Ivan Gianolla, Diretor Geral da Votocel pela autorização e apoio, aos Srs. Adilson Dorta Mariano, Aldo Arruda Mortara, José Adalberto Lopes e Dirceu Varejão pelo crédito na proposta de implantação da metodologia na Votocel. Também faz-se necessário um agradecimento especial ao Prof. Lin Chih Cheng, da UFMG, pela possibilidade de aprendizado proporcionada no acompanhamento do trabalho e outras pessoas que, direta ou indiretamente, colaboraram na execução desse trabalho.

\section{Referências Bibliográficas}

1. Akao, J. and Ohfuji, T. - "Recent Aspects of Quality Function Deployment in Services Industries in Japan", in: 
Proceedings of the International Conference on Quality Control, p. 17-26, Rio de Janeiro (1989).

2. Rosati, A. C. - "DFM e QFD Aplicados aos Sistemas de Distribuição de Energia e Sinal Automotivos (Chicotes Elétricos)", in: Anais do IX Simpósio de Engenharia Automotiva - SIMEA, p. 395-404, São Paulo (1997).

3. Fragoso, H. R. - "O Ciclo de Desenvolvimento de Produtos da Volkswagen Caminhões e Ônibus", in: Anais do $1^{\circ}$ Congresso Brasileiro de Gestão de Desenvolvimento de Produtos, p. 25-36, Belo Horizonte - MG, ago (1999).

4. Nogueira, T. M. et al. - "Quality Assurance: An Application of QFD to the Production Startup of a New Engine Line", in: Proceedings of the 5th International Symposium on Quality Function Deployment, p. 2683, Belo Horizonte - MG, ago (1999).

5. Santiago, L. P. \& Cheng, L. C. - "Improving the Product Development System of Auto Suppliers Using the QFD Method", in: Proceedings of the 5th International Symposium on Quality Function Deployment, p. 182-191, Belo Horizonte - MG, ago (1999).

6. Hashimoto, T. et al. - "Implantação do QFD na Caraíba Metais”. Gestão da Qualidade Total em Serviços Casos Reais. Fundação Christiano Ottoni, Belo Horizonte (1996).

7. Azambuja, M. S. \& Godoy, L. P. - "Quality Program Implementation in a Hotel Chain", in: Proceedings of the 15th International Conference on CAD/CAM, Robotics \& Factories of the Future, Águas de Lindóia - SP, ago (1999).

8. Passeto, S. C.; Echeveste, M. E. \& Ribeiro, J. L. D. "Desdobramento da Qualidade em Serviços: Melhorando o Desempenho de uma Academia de Ginástica", in: Anais do 1 Congresso Brasileiro de Gestão de Desenvolvimento de Produtos, p. 167-176, Belo Horizonte - MG, ago (1999).

9. Hirai, P. et al. - "O QFD no Processo de Desenvolvimento de um Produto de Previdência Privada na BrasilPrev", in: Anais do 1ํ Congresso Brasileiro de Gestão de Desenvolvimento de Produtos, p. 67-78, Belo Horizonte - MG, ago (1999).

10. Moysés, G. L. R. \& Turrioni, J. B. - "Análise da Utilização do QFD no Setor de Serviços: Aplicação em um
Sistema de Ensino", in: Anais do 2 Congresso Brasileiro de Gestão de Desenvolvimento de Produtos, p. 326-333, São Carlos - SP, ago (2000).

11. Cheng, L. C. \& Sarantopoulos, I. A. - "QFD in Brazil: A Successful Diffusion Process into Organizations", in: Proceedings of the 1st International Symposium on Quality Function Deployment, p. 77-84, Tokyo, Japão (1995).

12. Ormanese, F. M. et al. - "Exploring a New Market Using QFD", in: Proceedings of the 8th Symposium on Quality Function Deployment, p. 1-10, Novi, MI, jun (1996).

13. Guedes, L. B. R. et al. - "Obtaining Countrywide Sucess through QFD Implementation in the Development Process of a Popular Brazilian Food Product", in: Proceedings of the 5th International Symposium on Quality Function Deployment, p. 67-74, Belo Horizonte MG, ago (1999).

14. Cabral, P. et al. - "Adding Value to the Packaging Development Guided by QFD”, in: Proceedings of the 5th International Symposium on Quality Function Deployment, p. 75-82, Belo Horizonte - MG, ago (1999).

15. Yeung, V. W. S. \& Lau, K. H. - "Injection Moulding, 'CMOLD'CAE Package, Process Parametrer Design and Quality Function Deployment: A Case Study of Intelligent Materials Processing”, in: Journal of Materials Processing Technology, Vol. 63, No. 1-3, p. 481-487, Jan (1997).

16. Cheng, L. C. et al. - "QFD - Planejamento da Qualidade". Editora Líttera Maciel Ltda., Belo Horizonte (1995).

17. Akao, Y. "Quality Function Deployment - QFD - Integrating Customer Requirements into Product Design". Edited by Y. Akao, Productive Press, Portland, Oregon (1990).

18. Miguel, P. A. C. - "Qualidade: Enfoques e Ferramentas". Editora Artliber, São Paulo (2001).

19. Mortara, A. - "Filmes de Poliprileno Biaxialmente Orientados - Evolução e Fexibilidade sem Limites". Informação Técnica Votocel, Votorantin, jun (2000).

20. Ohfuji, T., Ono, M. \& Akao, Y. "Métodos de Desdobramento da Função Qualidade”, Volume 1. Fundação Christiano Ottoni, Belo Horizonte (1997).

Recebido:23/11/01 Aprovado: 09/12/02 